I N S T I T U T O

$\mathrm{DE}$

M E D I C I N A

T R O P I C A L

$\mathrm{DE}$

S ÃO PAULO

JOURNAL OF THE SÃO PAULO INSTITUTE OF TROPICAL MEDICINE

${ }^{1}$ Universidade de São Paulo, Faculdade de Odontologia, Departamento de Estomatologia, São Paulo, São Paulo, Brazil

2Universidade de São Paulo, Instituto de Medicina Tropical de São Paulo, Laboratório de Virologia (LIM 52), São Paulo, São Paulo, Brazil

Correspondence to: Paulo Henrique BrazSilva

Universidade de São Paulo, Instituto de Medicina Tropical de São Paulo, Laboratório de Virologia (LIM 52), Av. Dr. Enéas Carvalho de Aguiar, 470, CEP 05403-000, São Paulo, SP, Brazil

Tel: +55 11 3061-7066

E-mail: pbraz@usp.br

Received: 5 August 2019

Accepted: 16 September 2019
http://doi.org/10.1590/S1678-9946201961054

\section{Unusual presentation of a disseminated oral HPV infection after combined antiretroviral therapy initiation}

Daniela Assis do Vale ${ }^{1}$, Lais Magda Araújo Ferracini' ${ }^{1}$, Marcus Vinícius da Silva Rodrigues Bueno', Ana Carolina Mamana Fernandes Souza², Paulo Henrique Braz-Silva ${ }^{\circledR 1,2}$, Karem López Ortega ${ }^{(1)}$

\section{ABSTRACT}

HPV clinical manifestations have their characteristics modified by the use of combined antiretroviral therapy (cART), although its incidence is unaffected by cART. We report an unusual presentation of oral HPV infection and discuss an effective treatment for disseminated HPV lesions. A 52-year-old male of Asian-origin, HIV-seropositive, presented with extensive nodular lesions throughout the oral mucosa extending to the oropharyngeal region. Biopsy followed by histopathological examination and HPV genotyping were performed. The treatment was initiated with topical application of podophyllin and trichloroacetic acid. HPV lesions in oral mucosa are generally easy to handle. Extensive lesions can make it difficult to choose an effective treatment that meets the patient's particularities and medication availability.

KEYWORDS: HIV. HPV. Condyloma acuminate. Focal epithelial hyperplasia.

\section{INTRODUCTION}

Combined antiretroviral therapy (cART) has increased life expectancy and reduced the incidence of most opportunistic infections in patients infected by the human immunodeficiency virus (HIV). Although human papillomaviruses (HPV) clinical manifestations can have their characteristics modified, their incidence is not affected by cART ${ }^{1}$.

HPV is involved in a variety of oral lesions as common warts, condyloma acuminata, squamous cells papilloma and focal epithelial hyperplasia. All these presentations are benign, asymptomatic, exophytic, slow grown lesions ${ }^{2,3}$.

Cytodestructive and excisional methods or their combination have been the most common choices for a low-cost treatment of lesions caused by HPV ${ }^{4,5}$. The first option of treatment for HPV oral lesions is almost always their surgical removal.

\section{CASE REPORT}

A 52-year-old male of Asian-origin was referred for the diagnosis of multiple lesions in the oral cavity. The patient reported to be HIV-seropositive for 12 years, during which he did not follow the antiretroviral treatment adequately as he took medications on an intermittent basis. When the oral lesions first appeared, T CD4+ was 70 cells $/ \mathrm{mm}^{3}$, and the patient changed his behaviour and adhered to the proposed treatment (Lamivudine, Tenofovir and Atazanavir). Four months after cART was initiated, the patient had significant improvement in the immune system 
and an increase in T CD4+ to 486 cells $/ \mathrm{mm}^{3}$ together with undetectable viral load. Concomitantly, the patient observed an marked increase in the amount and size of the oral lesions, which began to be aesthetically and functionally inconvenient, even for daily routine activities, such as mastication. After four years living with the increase in the amount of oral mucosa lesions, the patient sought treatment.

The intraoral clinical examination detected extensive nodular lesions on the entire oral mucosa (i.e. palate, tongue, mouth floor, and alveolar, jugal and labial mucosa), extending to the oropharyngeal region. The lesions were asymptomatic, whitish and erythematous. The majority of the lesions, like those seen in the palate and tongue, were sessile and had a smooth surface, whereas the others were pedunculated and had papillary projections (Figures 1, 2 and 3).

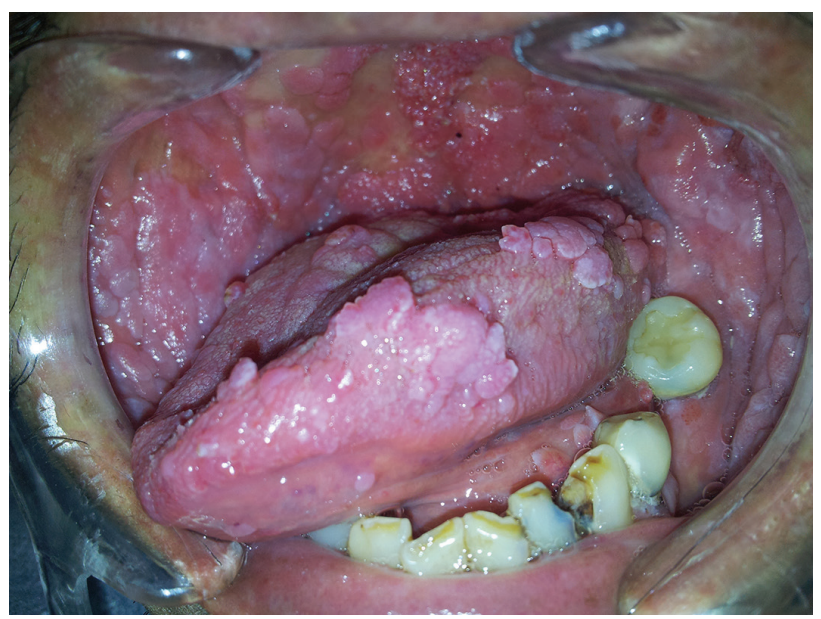

Figure 1 - View of the lateral edge and the dorsum of the tongue showing pedunculated nodules with smooth surface and lighter color than the mucosa.

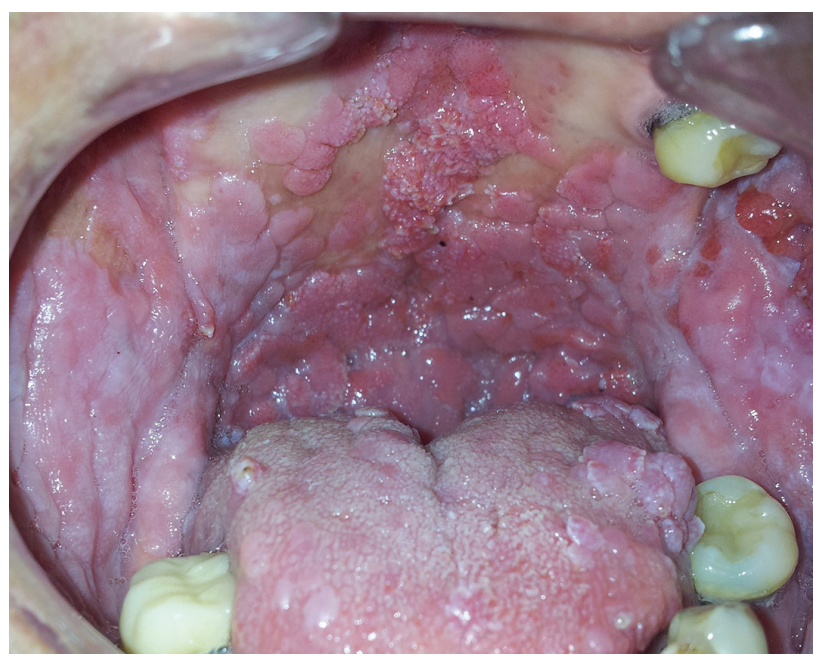

Figure 2 - View of the bilateral jugal mucosa and the soft palate showing multiple sessile nodules and some projections, resulting in a cobblestone appearance. In the hard palate, one can observe some reddish papillomatous lesions.

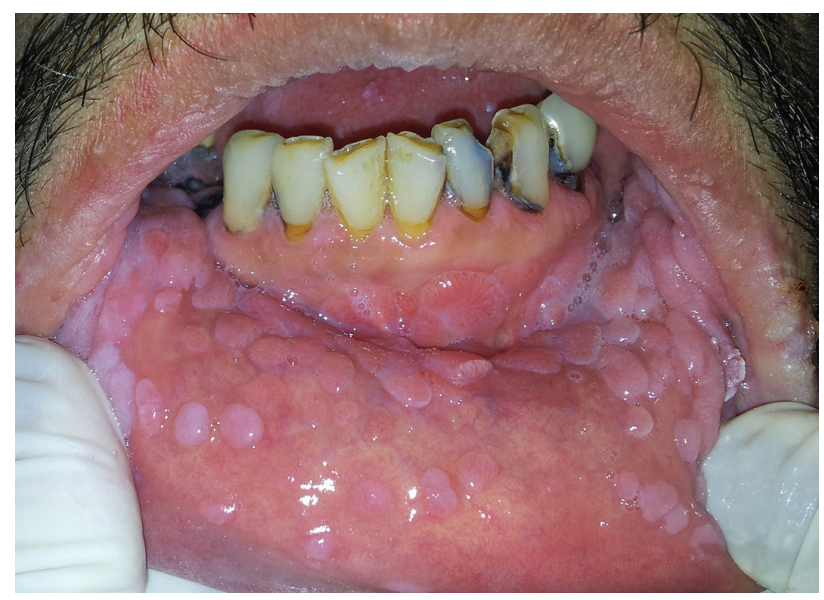

Figure 3 - View of flat lesions with smooth surface on the lower labial mucosa, gingival sulcus.

Incisional biopsy was performed and the material obtained was sent for histopathological examination. The sections were stained with hematoxylin and eosin, revealing a mucosal fragment covered with parakeratinised stratified squamous epithelium and acanthosis areas forming either blunt or sharp projections, giving a papillomatous aspect to the epithelium. At higher magnification, one could observe the formation of micro-abscesses, clusters of neutrophils and diffuse presence of koilocyte-like cells (Figure 4). In addition, a tissue sample was submitted to DNA extraction and analyzed by INNO-Lipa HPV genotyping extra II test (INNO-Lipa Innogenetics N.V., Ghent, Belgium), as well as PCR sequencing with generic primers, revealing the presence of HPV-32.

Based on the clinical aspect of the lesions, histopathological findings and detection of HPV-32, it was possible to diagnose the presence of epithelial proliferation due to HPV infection. The unusual clinical presentation raised discussion on the lesions, suggesting that it might be

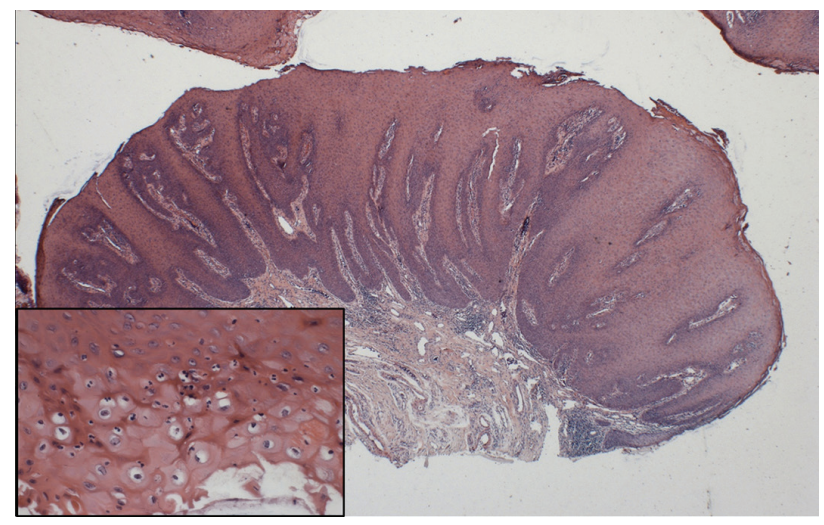

Figure 4 - Histopathological examination of the lesion showing epithelial proliferation forming projections towards the connective tissue. In detail, epithelial cells showing a clear perinuclear halo compatible with koilocytosis. 
a case of either focal epithelial hyperplasia (FEH), a lesion strongly associated with HPV- $32^{4}$, or condyloma acuminata aggravated by the syndrome of immune reconstitution (SIR).

Due to the extension of lesions in the patient and, consequently, the great number of surgical procedures that would be necessary, as well as the high viral infectivity rate (leading to the possibility of re-contamination of the mucosa during manipulation), treatment based on surgical removal was discarded. The treatment was initiated with topical application of podophyllin and trichloroacetic $\operatorname{acid}^{6,7}$. Nevertheless, the long-term treatment unfortunately made adhesion difficult for the patient, who did not attend the appointments after some sessions.

\section{DISCUSSION}

Focal epithelial hyperplasia and oral condyloma acuminata have similar clinical and histopathological characteristics. Both are caused by low-risk HPVs, with condyloma being more often associated with HPV-6 and HPV-11, although several other types (including HPV-32 and more rarely high-risk HPVs) have been described in these lesions ${ }^{4,8,9}$.

The syndrome of immune reconstitution (SIR) is a significant clinical complication in HIV-infected patients, whose immune system improve quickly. Its paradoxical form is related to previously-treated opportunistic infections re-emerging after immune reconstitution resulting from antiretroviral therapy. The unmasking form occurs when an underlying or untreated opportunistic infection induces the recovering immune system to react against these pathogens. The exact mechanism leading to the syndrome of immune reconstitution is not yet clear, but some important factors are related to its emergence, such as the rapid immune recovery after initiating cART and/or rapid decline in viral load. In addition to these factors, the diagnosis is also based on the relationship between the beginning of antiretroviral therapy, the emergence of the disease (within about 3 months) and the clinical course of the opportunistic infection $^{10}$.

Based on these concepts, it is possible to speculate that the present case may be related to SIR, as the patient had a remarkable improvement in the immune response four months after cART in view of the clinical picture of unusual lesions caused by HPV.

There is no consensus in the literature regarding the treatment of extensive lesions as those reported here. However, because the viral load of HPV is much higher in patients with HPV-32 and multiple oral lesions ${ }^{11}$, it is natural to think that the use of medications acting locally and systemically would be the best option in case of viral proliferation. Anti-mitotic (e.g. cidofovir) and immunemodulating agents (e.g. imiquimod, interferon and cyclophosphamide) ${ }^{6}$ have been used with good results. Treatment with these medications were discarded because of their high cost and the lack of consistent evidence-based recommendations.

HPV lesions in oral mucosa have low incidence and are generally easy to handle, with surgical excision being the treatment of choice. When these lesions affect a patient who has immunological deficiencies, one should intervene as soon as possible to prevent them from becoming extensive, making it difficult to choose an effective treatment. On the other hand, it is important to emphasize that the treatment should be chosen based on the patient's particularities (e.g. likelihood to adhere to treatment) and availability of medications. In addition to the accurate diagnosis, it is important to investigate the sub-type of HPV present in the patient for the prognosis and management of lesions.

\section{ACKNOWLEDGMENTS}

This work was supported by the Sao Paulo Research Foundation - FAPESP, Sao Paulo, Brazil (grant $\left.\mathrm{N}^{\circ} 2015 / 07727-9\right)$.

\section{REFERENCES}

1. Shiboski CH, Lee A, Chen H, Webster-Cyriaque J, Seaman T, Landovitz RJ, et al. Human papillomavirus infection in the oral cavity of HIV patients is not reduced by initiating antiretroviral therapy. AIDS. 2016;30:1573-82.

2. Anaya-Saavedra G, Flores-Moreno B, García-Carrancá A, Irigoyen-Camacho E, Guido-Jiménez M, Ramírez-Amador V. HPV oral lesions in HIV-infected patients: the impact of long-term HAART. J Oral Pathol Med. 2013;42:443-9.

3. Syrjänen S. Human papillomavirus infections and oral tumors. Med Microbiol Immunol. 2003;192:123-8.

4. Gemigniani F, Hernández-Losa J, Ferrer B, García-Patos V. Focal epithelial hyperplasia by human papillomavirus (HPV)32 misdiagnosed as HPV-16 and treated with combination of retinoids, imiquimod and quadrivalent HPV vaccine. J Dermatol. 2015;42:1172-5.

5. Scheinfeld N, Lehman DS. An evidence-based review of medical and surgical treatments of genital warts. Dermatol Online J. 2006;12:5.

6. Ramírez-Fort MK, Au SC, Javed SA, Loo DS. Management of cutaneous human papillomavirus infection: pharmacotherapies. Curr Probl Dermatol. 2014;45:175-85.

7. Carmona Lorduy M, Harris Ricardo J, Hernández Arenas Y, Medina Carmona W. Use of trichloroacetic acid for 
management of oral lesions caused by human papillomavirus. Gen Dent. 2018;66:47-9.

8. Khanal S, Cole ET, Joh J, Ghim SJ, Jenson Ab, Rai SN, et al. Human papillomavirus detection in histologic samples of multifocal epithelial hyperplasia: a novel demographic presentation. Oral Surg Oral Med Oral Pathol Oral Radiol. 2015;120:733-43.

9. Syrjänen SM, Syrjänen KJ, Happonen RP, Lamberg MA. In situ DNA hybridization analysis of human papillomavirus (HPV) sequences in benign oral mucosal lesions. Arch Dermatol Res. 1987;279:543-9.

10. Martin-Blondel G, Mars LT, Liblau RS. Pathogenesis of the immune reconstitution inflammatory syndrome in HIV-infected patients. Curr Opin Infect Dis. 2012;25:312-20.

11. Camacho-Aguilar S, Ramírez-Amador V, Rosendo-Chalma P, Guido-Jiménez M, García-Carrancá A, Anaya-Saavedra G. Human papillomavirus load in benign HPV-associated oral lesions from HIV/AIDS individuals. Oral Dis. 2018;24:210-4. 